\title{
Effect of a Magnetic Field on an Atomic Orbital
}

\author{
Sami M. AL-Jaber ${ }^{1}$, AbdelRahman M. Abu-Labdeh ${ }^{2}$ \\ ${ }^{1}$ Department of Physics, An-Najah National University, Nablus, Palestine \\ ${ }^{2}$ Department of Physics, AAUJ, Jenin, Palestine \\ E-mail: jaber@najah.edu, alabdeh@aauj.edu
}

Received December 16, 2010; revised December 18, 2010; accepted December 22, 2010

\begin{abstract}
We consider the effect of a magnetic field on the motion of an atomic electron in its orbit. The usual treatment deals with the change in magnetic dipole moment assuming the electron's speed changes but the radius of its orbit remains unchanged. We derive the change in the magnetic dipole moment allowing both the speed and the radius to change. The cases of fixed radius on one hand and of fixed speed on the other are treated as special cases of our general case.
\end{abstract}

Keywords: Diamagnetism, Effect of Magnetic Field on Atomic Orbital, Magnetic Dipole Moment of an Orbital Electron

\section{Introduction}

Typically, classical electromagnetism predicts a change in the magnetic dipole moment of an orbital electron when an external magnetic field is set up normal to the plane of the electron's orbit [1, 2]. It is usually assumed that the speed of the electron changes but the radius remains unchanged during the time through which the magnetic field is changing [3]. This assumption is not consistent with the effect of perturbation on classical orbits for Coulomb potentials. Griffiths [3] discussed the effect of a magnetic field on an atomic orbital and derived the change in the magnetic dipole moment of the electron keeping the orbital radius unchanged. He also mentioned (without derivation) that if one assumes constant speed while the radius changes then he would get a change in the magnetic moment which is twice its magnitude for the case of fixed radius and change in speed. When an atomic electron is subject to an external magnetic field, most authors attribute the speed change to the additional magnetic force. This justification is confusing since the students learned that magnetic forces cannot do work and thus incapable to change the electron's speed. A more thoughtful idea is to attribute the speed change to the modified centripetal force and to assert a fixed radius. Even though there has been considerable interest in the effect of magnetic field on the motion of an electron [4-8], but there has been no direct investigation of the above problem for the general case in which both the speed and the radius are allowed to change. Therefore in the present paper we discuss the effect of an external magnetic field on an atomic orbital electron. In particular, we derive the change in the electron's magnetic moment for the general case in which both the speed and the orbit radius are allowed to change during the time through which the magnetic field is increasing to its final value. Our treatment for the general case is motivated not only by its fundamental importance but its close relation to the so-called satellite paradox which deals with the effect of atmospheric drag on a satellite orbit [9-11].

\section{An Atomic Electron in a Magnetic Field}

Consider an atomic electron that rotates counterclockwise with a speed $v_{0}$ in a circular orbit, in the x-y plane, of radius $r_{0}$. In practice the period of the electron's motion $T=2 \pi r_{0} / v_{0}$ is so small that one may assume it constitutes a steady current:

$$
I=\frac{e}{T}=\frac{e v_{0}}{2 \pi r_{0}}
$$

and thus its orbital dipole moment is

$$
\vec{\mu}=I \pi r_{0}^{2}=-\frac{1}{2} e v_{0} r_{0} \hat{z}
$$

where $-e$ is the charge of the electron. The electric force exerted on the electron by the nucleus (of charge $+e$ ) is balanced by the centripetal force,

$$
\frac{1}{4 \pi \varepsilon_{0}} \frac{e^{2}}{r_{0}^{2}}=m \frac{v_{0}^{2}}{r_{0}}
$$


where $m$ is the mass of the electron. Now, introduce a magnetic field in the $z$ direction, of strength $B(t)$, which, initially zero, increases to $B$. The form of $B(t)$ is irrelevant provided that it varies sufficiently slowly, which means on a time scale long compared to the electron's orbital period. The magnetic force exerted on the electron points toward the center and of magnitude $e v B$, so the centripetal force must be modified to accommodate for magnetic force. Thus

$$
\frac{e^{2}}{4 \pi \varepsilon_{0} r^{2}}+e v B=\frac{m v^{2}}{r}
$$

where we allow both the speed and the radius of the orbit to change from $v_{0}$ to $v$ and $r_{0}$ to $r$ respectively. Letting $v=v_{0}+\Delta v$ and $r=r_{0}+\Delta r$, Equation (4) reads

$$
\frac{e^{2}}{4 \pi \varepsilon_{0}} \frac{1}{\left(r_{0}+\Delta r\right)^{2}}+e\left(v_{0}+\Delta v\right) B=\frac{m\left(v_{0}+\Delta v\right)^{2}}{\left(r_{0}+\Delta r\right)}
$$

which, with the help of Equation (3), can be written as

$$
\frac{m r_{0} v_{0}^{2}}{r_{0}^{2}} \frac{1}{\left(1+\frac{\Delta r}{r_{0}}\right)^{2}}+e B\left(v_{0}+\Delta v\right)=\frac{m\left(v_{0}+\Delta v\right)^{2}}{r_{0}\left(1+\frac{\Delta r}{r_{0}}\right)}
$$

Using binomial expansion to first order in $\Delta r$ and $\Delta v$ and neglecting terms containing the product $(\Delta r)(\Delta v)$, Equation (6) yields

$$
e B v_{0}\left(1+\frac{\Delta v}{v_{0}}\right)=\frac{m v_{0}^{2}}{r_{0}}\left(2 \frac{\Delta v}{v_{0}}+\frac{\Delta r}{r_{0}}\right)
$$

which, with a further use of binomial expansion for the left hand side term, gives

$$
e B v_{0}=\frac{m v_{0}^{2}}{r_{0}}\left(2 \frac{\Delta v}{v_{0}}+\frac{\Delta r}{r_{0}}\right)
$$

Our result in Equation (8) is the general case which allows changes in the speed and the radius of the orbit. Before we proceed further, we need to consider the two special cases in the following section.

\section{Special Cases}

Our aim here is utilize our result of Equation (8) for two special cases: the first deals with fixed orbit $(\Delta r=0)$ but $v$ changes and the second deals with fixed speed $(\Delta v=0)$ but $r$ changes.

\subsection{The Fixed Orbit Radius Case}

For $\Delta r=0$, Equation (8) immediately gives

$$
\Delta v=\frac{e B r_{0}}{2 m}
$$

which corresponds to a change in the magnetic dipole moment predicted by Equation (2), namely

$$
\Delta \mu=-\frac{1}{2} e r_{0} \Delta v=-\frac{e^{2} r_{0}^{2}}{4 m} B
$$

The above result is the same as that derived by Griffiths in ref. [3]. This change in magnetic moment corresponds to a change in the orbital angular momentum, $L$ through the relation $\vec{\mu}=-\frac{e}{2 m} \vec{L}$, with the result

$$
\Delta \vec{L}=-\frac{2 m}{e} \Delta \vec{\mu}=\frac{e r_{0}^{2}}{2} \vec{B}
$$

One may also derive $\Delta \vec{L}$ and thus $\Delta \vec{\mu}$ by considering the angular impulse exerted by the induced electric field which is created during the change of the magnetic field. Faraday's law of induction gives an induced electric field given by

$$
\vec{E}=-\frac{r_{0}}{2} \frac{d B}{d t} \hat{\phi}
$$

and thus the torque $\vec{\tau}$ exerted on the electron is

$$
\vec{\tau}=\vec{r} x(-e \vec{E})=\frac{e r_{0}^{2}}{2} \frac{d B}{d t} \hat{z}
$$

which immediately gives the change in angular momentum, that is

$$
\Delta \vec{L}=\int \vec{\tau} d t=\int_{0}^{B} \frac{e r_{0}^{2}}{2} \frac{d \vec{B}}{d t} d t=\frac{e r_{0}^{2}}{2} \vec{B}
$$

Therefore, upon using $\Delta \mu=-\frac{e}{2 m} \Delta L$, one gets the correct change in the magnetic moment given in Equation (10). It is interesting to calculate the work, $W$ done by the induced electric field:

$$
\begin{aligned}
W & =\int(-e \vec{E}) \cdot d \vec{S}=e \int \frac{r_{0}}{2} \frac{d B}{d t} d S \\
& =e \int_{0}^{B} \frac{r_{0}}{2} \bar{v} d B=\frac{e r_{0}}{2} B\left(v_{0}+\frac{1}{2} \Delta v\right),
\end{aligned}
$$

where we used for the average speed $\bar{v}=v_{0}+\frac{1}{2} \Delta v$. Using the expression for $\vec{\mu}$ in Equation (2) and that of $\Delta \vec{\mu}$ in Equation (10), the work done takes the form

$$
W=-\left(\vec{\mu}+\frac{1}{2} \Delta \vec{\mu}\right) \cdot \vec{B}
$$

Alternatively, one may calculate the change in the kinetic energy of the electron,

$$
\Delta K=K_{f}-K_{0}=\frac{1}{2} m\left(v_{0}+\Delta v\right)^{2}-\frac{1}{2} m v_{0}^{2}
$$




$$
=m\left(v_{0} \Delta v+\frac{1}{2}(\Delta v)^{2}\right)
$$

which upon the substitution of $\Delta v$ from Equation (9), $\vec{\mu}$ from Equation (2) and $\Delta \vec{\mu}$ from Equation (10), the change in the kinetic energy takes the form

$$
\Delta K=-\left(\vec{\mu}+\frac{1}{2} \Delta \vec{\mu}\right) \cdot \vec{B}
$$

The above result for $\Delta K$ is equal to the work done given in Equation (17) and thus the work-energy theorem is verified.

\subsection{The Fixed Speed Case}

For $\Delta v=0$, Equation (8) gives

$$
\Delta r=\frac{e B r_{0}^{2}}{m v_{0}}
$$

and in this case the change in the magnetic moment is given by

$$
\Delta \mu=-\frac{1}{2} e v_{0} \Delta r
$$

which upon using Equation (19) becomes

$$
\Delta \mu=-\frac{e^{2} r_{0}^{2}}{2 m} B
$$

Obviously, our result shows that the magnitude of the change of the magnetic moment for fixed speed is twice its magnitude for the fixed radius case. The result in Equation (21) is what Griffiths claimed in reference [3] but without any derivation. This change in the magnetic moment corresponds to a change in the angular momentum, that is

$$
\Delta \vec{L}=-\frac{2 m}{e} \Delta \vec{\mu}=e r_{0}^{2} \vec{B}
$$

Going back to the general case in which both the speed and the radius are changing, one may rewrite Equation (8) in the form

$$
\left(r_{0} \Delta v+v_{0} \Delta r\right)=\frac{e B r_{0}^{2}}{m}-r_{0} \Delta v
$$

In the present case, the variation in the magnetic moment is

$$
\Delta \mu=-\frac{1}{2} e\left(r_{0} \Delta v+v_{0} \Delta r\right)
$$

which upon the use of Equation (23) it becomes

$$
\Delta \mu=-\frac{1}{2} e\left(\frac{e B r_{0}^{2}}{m}-r_{0} \Delta v\right)=-\frac{e^{2} r_{0}^{2}}{2 m} B+\frac{1}{2} e r_{0} \Delta v .
$$

Note that the last term on the right hand-side looks like the same as for the fixed radius case. Therefore, using Equation (9), we get

$$
\Delta \mu=-\frac{e^{2} r_{0}^{2}}{2 m} B+\frac{e^{2} r_{0}^{2}}{4 m} B=-\frac{e^{2} r_{0}^{2}}{4 m} B
$$

The above result is exactly the same as that for the fixed radius case, and thus it corresponds to the same change in the angular momentum which is given in Equation (14).

\section{Conclusions}

In this work, the effect of an external magnetic field on an atomic electron has been examined. The usual treatment of this problem assumes (without justification) a fixed radius for the orbit but allow the electron's speed to change. In the present paper we considered the general case in which both the speed and the radius are allowed to change. We used binomial expansion to first order in the change of speed and in the change of the radius. The two special cases for fixed orbit and for fixed speed were deduced from our general case and in each of these two cases the change in the magnetic moment and the change in the angular momentum have been derived. Interestingly, our result for the general case yields a change in the magnetic moment which is the same as that for the fixed radius case.

\section{References}

[1] R. P. Feynman, R. B. Leighton and M. Sands, "The Feynman Lectures on Physics,” Vol. 2, Addison-Wesley, Reading, MA, 1964.

[2] E. M. Purcell, "Electricity and Magnetism," 2nd Edition, McGraw-Hill, New York, 1985.

[3] D. J. Griffiths, "Introduction to Electrodynamics," 3rd Edition, Prentice-Hall, New Jersey, 1999.

[4] N. Nauthier, “A New-Faraday Approach to Electromagnetic Energy and Angular Momentum Storage in an Electromechanical System," American Journal of Physics, Vol. 70, No. 10, 2002, p. 1034. doi:10.1119/1.1485715

[5] S. Olszewski and T. Rolinski, "Orbital Angular Momentum, Its Torque and Magnetic Moment Calculated for the Electrons Circulating on the Landau Levels of a Crystalline Solid,” Physica Status Solidi(b), Vol. 245, No. 8, 2008, pp. 1623-1634. doi:10.1002/pssb.200743381

[6] J. M. Aguirregabiria, A. Hernandez and M. Rivas, “A Lewis-Tolman - Like Paradox," European Journal of Physics, Vol. 3, No. 1, 1982, p. 30. doi:10.1088/0143-0807/3/1/008

[7] V. Kumar, "Understanding the Focusi Charge Particle in a Solenoid Magnetic Field," American Journal of Physics, Vol. 77, 2009, p. 737. 
doi:10.1119/1.3129242

[8] S. L. O’Dell and R. K. P. Zia, “Classical and Semiclassical Diamagnetism: A Critique of Treatment in Elementary Texts," American Journal of Physics, Vol. 54, No. 1, 1986, pp. 32-35. doi:10.1119/1.14766

[9] B. D. Mills, Jr., "Satellite Paradox," American Journal of Physics, Vol. 27, No. 2, 1959, pp. 115-117. doi:10.1119/1.1934766

[10] L. Blitzer, "Satellite Orbit Paradox: A General View," American Journal of Physics, Vol. 39, No. 8, 1971, pp. 882-886. doi:10.1119/1.1986317

[11] F. P. J. Rimrott and F. A. Salustri, "Open Orbits in Satellite Dynamics,” Technische Mechanik, Vol. 21, No. 3, 2001, pp. 207-214. 